\title{
CASE REPORT ON MANAGEMENT OF ONE ELBW BABY IN A LEVEL II NICU IN KIMS AMLAPURAM
}

Lalat Barun Patra1, Sindhura Kasturi², Md. Jakeer Shaik ${ }^{3}$

\section{HOW TO CITE THIS ARTICLE:}

Lalat Barun Patra, Sindhura Kasturi, Md. Jakeer Shaik. "Case Report on Management of One ELBW Baby in a Level II NICU in KIMS Amlapuram". Journal of Evolution of Medical and Dental Sciences 2015; Vol. 4, Issue 49, June 18; Page: 8615-8618, DOI:10.14260/jemds/2015/1247

ABSTRACT: INTRODUCTION: The extremely low birth weight (ELBW) premature baby is an infant born at 1000 grams or less (2pounds 3ounces), generally before 28 weeks gestation, sometimes referred to colloquially as microprimies. These infants present one of the greatest medical and ethical challenges to the field of pediatrics. These subpopulation of neonates require extra vigilance by the primary health care provider with proper attention to medical sequelae and subsequent development. With the availability of neonatal intensive care, the outcomes of high-risk infants born either preterm or with serious medical or surgical conditions have been improved [1,2] Many of these improvements can be attributed to the concept and implementation of regionalized systems of neonatal care. Survival of these Extremely low birth weight babies can be attributed to the widespread use of surfactants, maternal steroids, and advancements in neonatal technologies like CPAP, ventilators, I NO and ECMO.

CASE REPORT: A 23 year second gravida mother with uneventful antenatal history came to KIMS hospital with labour pain and leaking $\mathrm{p} / \mathrm{v}$ for safe institutional delivery. She was diagnosed to have twin pregnancy by antenatal scan in first trimester. At the time of presentation her gestational age was 26weeks 4 days according to her last menstrual period. One dose of betamethasone was given. A 870 gms female child (twin 1) was delivered through normal vaginal delivery. Child was vigorous at birth with no need of any resuscitation. After delivery of first twin mother was shifted for cesarean section in view of non-reactive CTG. Twin II was declared as intra uterine death.

Twin I maintained vitals and blood glucose levels. Prophylactic antibiotic (Piperacillin and tazobactum plus amikacin) started along with maintenance fluids. Baby showed signs of respiratory distress since birth. Decision was made to administer surfactant immediately. Chest X-ray at 6hrs of life showed changes significant of hyaline membrane disease. Due to non-availability of surfactant there was a delay and surfactant could be given at $12 \mathrm{hrs}$ of life. No complications were noted post surfactant administration. Signs of respiratory distress got settled within $24 \mathrm{hrs}$ of life. Umbilical vein was catheterised.

Baby was carefully nursed in a normothermic environment and blood sugar and electrolyte values were monitored regularly. Supplementation of micro and macro nutrients were maintained throughout. Initial Sepsis screen results were negative.

Hyperbilirubinemia without ABO incompatability or signs of hemolysis noted on day 3 of life was managed with single surface phototherapy for $48 \mathrm{hrs}$.

Baby was planned to be kept on total parental nutrition but due to non-availability TPN could not be instituted. Feeding was started in form of expressed breast milk through orogastric tube on day 3 of life and gradually increased based on tolerability of the child. Throughout baby was monitored for signs of NEC. HMF was added to breast milk to increase the calorie density.

On day 7 of life umbilical artery and venous catheter were removed. 
On day 9 of life baby developed episodes of apnea with bradycardia and cyanosis but responsive to stimulation. Caffeine loading was given followed by maintenance dose and antibiotics were upgraded. Multiple episodes of apnea recurred in a period of $24 \mathrm{hrs}$ during which baby was carefully monitored and stimulated. Child was apnea free after that episode. Caffeine was discontinued at 35 weeks of postnatal age.

Nonnutritive sucking was started when child attained 31 wks of gestation followed by orogastric tube feeding. Spoon feed were introduced at $32 \mathrm{wks}$ of gestational age with gradual increase in quantity with exclusive spoon feeds achieved at 34wks of gestational age.

On day 20 of life holosystolic murmur suggestive of PDA was heard on auscultation with no signs of cardiac failure. Three doses of Ibuprofen administered followed by disappearance of murmur with echocardiographic evidence of PDA closure.

Neurosonogram on day 7 and 30, ROP screening at 5wks postnatal period and 2D echo all showed normal study.

Baby was immunized at 6 weeks of postnatal period with BCG, OPV zero dose.

Baby attained birth weight at 30 days of life. Weight gain has been documented and baby discharged with weight of $1.3 \mathrm{kgs}$ at 36 weeks of postnatal age.

DISCUSSION: Respiratory distress syndrome, Apnea of prematurity, Feeding intolerance, Necrotizing enterocolitis Growth failure, infection, Intraventricular hemorrhage, Hearing loss, Retinopathy of prematurity, Patent ductus arteriosus, Anemia of prematurity are the major Problems of Extremely Low Birth weight Infants.

Respiratory distress syndrome (RDS), or hyaline membrane disease (HMD), has been recognized as the most common complication of prematurity, with more than half of those with a birth weight of between 501grams and 1500 grams showing signs of RDS.(3,4) In infants with RDS, exogenous surfactant has been shown to reduce mortality and any form of pulmonary air leaks by about $30 \%$ and $50 \%$, respectively. Though early surfactant is recommended due to unavailability in our setup a delay of $12 \mathrm{hrs}$ is present, but baby showed decreased signs of distress after surfactant.

Sepsis is most important cause of morbidity and mortality in preterm and low birth weight babies. In our case prophylactic antibiotics were started as child has risk factors for sepsis. According to our protocol piperacillin and tazobactum along with amikacin are started. Later, on day 7 screen came positive along with repeated episodes of apnea hence antibiotics upgraded to meropenem and amikacin.

A persistently patent ductus arteriosus (PDA) in preterm infants can have significant clinical consequences especially in very immature infants with extremely low birth weight $(<1000 \mathrm{~g})$. On the basis of the current clinical data, there are no benefits and possibly harm with early use of ibuprofen (Prophylaxis, Treatment) in the first 24 hours of life (Eg, pulmonary hypertension[5,6,7]) hence we intervened after appearance of clinical signs of PDA.

These infants who are usually born before 32-34 weeks gestation have inadequate body stores of most of the nutrients. Expressed breast milk has inadequate amounts of protein, energy, calcium, phosphorus, trace elements (Iron, zinc) and vitamins (D, E \& K) that are unable to meet their daily recommended intakes. Hence, these infants need multi-nutrient supplementation till they reach term gestation (40 weeks postmenstrual age).[8] 
ELBW infants are at increased risk for both neurosensory hearing loss and conductive hearing loss. All ELBW infants need to have brainstem auditory-evoked response hearing screening performed before 1 month corrected age (If not at discharge). Further follow-up should be as recommended by the audiologist or whenever there is any clinical or parental concern.[9]

NEC affects $1-3 \%$ of all neonatal intensive care unit (NICU) admissions and about $10-15 \%$ of ELBW infants with a mortality rate between $10-50 \%$. Changes in feeding practices for VLBW babies, including encouraging use of human breast milk and applying specific feeding protocols with careful attention to the rate of advancement of feeds, could potentially decrease the incidence of this disease. Currently available evidence suggests that the use of probiotics may significantly decrease the incidence of NEC and mortality in VLBW newborns.

CONCLUSION: Evidence suggests that infants who are born at $<32$ weeks' gestation, weight $<1500 \mathrm{~g}$ at birth, or have medical or surgical complication, should be cared at a level III nursery with facilities for advanced respiratory support and well organized monitoring system, round the clock laboratory and imaging facilities, nutrition and pharmacy support with neonatal expertise, social services.[14] Though our set up is of level II nursery proper vigilance and regular monitoring helped in providing optimal care to the baby. Due to non-availability of surfactant in this area there is a delay of $12 \mathrm{hrs}$ in administration of surfactant. Even though our case is a candidate for TPN non availability of the preparations prevented us from starting it. We had to depend on glucose, electrolyte mixed fluids with a close watch over the possible disturbances of both and carefully increased breast milk through orogastric feeding till the baby could accept direct breast feeding. Due to which there is documentation of slow growth of child and baby reached its birth weight by day 30 of life. In spite of these drawbacks proper nursing care, supply of adequate nutrition and supplements, careful watch and appropriate management of complications, regular screening helped us to manage our case.

\section{REFERENCES:}

1. Mac Dorman MF, Kirmeyer S Fetal and perinatal mortality, United States, 2005. Natl Vital Stat Rep. 2009; 57(8): 1-19. Medline Google Scholar.

2. Clement MS Perinatal care in Arizona 1950-2002: a study of the positive impact of technology, regionalization and the Arizona perinatal trust. J Perinatol. 2005; 25(8): 503-508 Cross Ref Medline Google Scholar.

3. Hack M, Fanar off AA. Outcomes of extremely-low-birth-weight infants between 1982 and 1988. New England Journal of Medicine 1989; 321: 1642-1647.

4. Hack M, Horbar JD, Malloy MH, Tyson JE, Wright E, Wright L. Very low birth weight outcomes of the National Institute of Child Health and Human 8 development neonatal network.Pediatrics 1991;87:587-597

5. Gournay V, 2.Savagner C, 3.Thiriez G, 4.Kuster A, 5.Roze JC. Pulmonary hypertension after ibuprofen prophylaxis in very preterm infants. Lancet. 2002; 359(9316): 1486-1488. Cross Ref Medline Web of Science Google Scholar.

6. Gournay V, 2. Roze JC, 3. Kuster A, 4.et al. Prophylactic ibuprofen versus placebo in very prematureinfants: arandomised, double-blind, placebo- controlledtrial. Lancet. 2004; 364(9449): 1939-1944 Cross Ref Medline Web of Science Google Scholar.

7. Shah SS, 2.Ohlsson A Ibuprofen for the prevention of patent ductus arteriosus in preterm and/or low birth weight infants. Cochrane Database Syst Rev. 2006; (1): CD004213. 


\section{CASE REPORT}

8. AIIMS- NICU protocols 2008 Feeding of Low Birth weight Infants.

9. Critical Elements of Care for the Low Birth Weight Neonatal Intensive Care Unit (NICU) Graduate (CEC - LBW).

\section{AUTHORS:}

1. Lalat Barun Patra

2. Sindhura Kasturi

3. Md. Jakeer Shaik

\section{PARTICULARS OF CONTRIBUTORS:}

1. Assistant Professor, Department of Paediatrics, KIMS.

2. $3^{\text {rd }}$ Year Post Graduate, Department of Paediatrics, KIMS.

FINANCIAL OR OTHER COMPETING INTERESTS: None
3. $3^{\text {rd }}$ Year Post Graduate, Department of Paediatrics, KIMS.

\section{NAME ADDRESS EMAIL ID OF THE} CORRESPONDING AUTHOR:

Dr. Lalat Barun Patra,

Qr. No. 212, Godavari Block, KIMS Campus, Amlapuram-533201,

East Godavari.

E-mail: drlalatbarun@gmail.com

Date of Submission: 22/05/2015.

Date of Peer Review: 25/05/2015.

Date of Acceptance: 11/06/2015.

Date of Publishing: 18/06/2015. 